\title{
Research of Network Transient Performance at Small Perturbations
}

\author{
T. A. Makhkamov, A. M. Mirzabaev \\ National Research University: Moscow Power Engineering Institute, Moscow, Russia, \\ Tashkent State Technical University, Tashkent, Uzbekistan \\ Email: temur.ma@gmail.com, solarmir@mail.ru
}

Received February, 2013

\begin{abstract}
The advisability of the use of matrix methods of equations rearrangement for the investigated system which allows writing a secular equation is considered in this article. This approach greatly simplifies the analysis of performance of transient response in complicated multi-coupled electrical system at small perturbations.
\end{abstract}

Keywords: Stability; Process Control Performance Factors; Transient Response; Matrix Analysis; Coefficient Matrix; Secular Equation

\section{Introduction}

The stability is necessary but an insufficient condition of automatic control systems operability [1]. The control system stability means only that there is a decaying of the transient response in the system under the influence of external control or perturbation action. Upon that, a process decaying time, maximum deviation of controllable value and number of oscillations in the system are not defined, however, these values are very important process control performance factors.

Process control performance factors can be defined by means of various methods. First of all, they comprise transient response design by the set closed-loop transfer functions, definition of performance factors by a disposition of zeroes and poles, integral performance criteria, frequency-domain performance estimation and frequency-domain methods of transient response design [1-3].

\section{Analysis}

In case of electrical power system, the differential equation system describing processes in such system are linear (linearized) and look like in the matrix form [2]:

$$
\dot{x}=A \cdot x,
$$

where

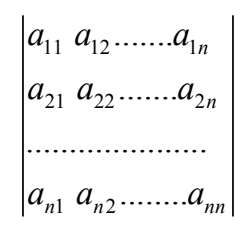

and $x=\left[x_{1}, x_{2}, \ldots, x_{n}\right]-$ a column matrix comprising deviations of required parameters of electrical power system condition.

The problems listed above become insuperable in cases of the systems described by equations with high degrees, i.e. complicated or multi-coupled systems. A primal problem is deriving of a secular equation for the investigated system. In this connection it is expedient to use matrix methods of rearrangement of the equations for the investigated system, then to receive a coefficient matrix for the differential equations $\mathrm{A}$ and further to set a secular equation under known algorithms of its setting. It is rather effective to apply Boher formulas [4] which generate factors of a secular equation of the investigated system. Let's consider an algorithm of generation of secular equation coefficients by a known matrix of coefficients of a matrix A.

Product of characteristic numbers of a square matrix A is equal to a determinant of this matrix. Let's notice that in case of equality to zero of any characteristic number the matrix $\mathrm{A}$ is singular $[5,7]$.

The sum of diagonal elements of a square matrix is equal to the sum of its characteristic numbers. Considering importance of this property, the special title, namely, a matrix trace is appropriated to the sum of diagonal elements of such matrix. This property is used to form coefficients of a secular equation for the investigated system.

Having designated the trace $A_{\kappa}$ (of the matrix A multiplied k times by itself) by $T_{\kappa}$ it is possible to write the useful recurrence formula expressing coefficients of a secular equation in terms of various $T_{K}$, so: 


$$
\begin{gathered}
a_{1}=-T_{1}, \\
a_{2}=-(1 / 2)\left(a_{1} T_{1}+T_{2}\right), \\
a_{3}=-(1 / 3)\left(a_{2} T_{1}+a_{l} T_{2}+T_{3}\right), \\
a_{n}=-(1 / n)\left(a_{n-1} T_{1}+a_{n-2} T_{2}+\ldots+a_{1} T_{n-1}+T_{n}\right) .
\end{gathered}
$$

This formula makes possible to define a secular equation diversely. It is obvious that it is rather effective for algorithmization of determination of a secular equation's coefficients.

Let's apply algorithm (3) to model of an electric system (electrical power system) which has $n=6$ in the case of availability of a strong excitation on synchronous generator with automatic excitation control; the matrix of differential equation coefficients for the system looks like:

$$
A=\left|\begin{array}{lllllc}
0 & a_{12} & 0 & 0 & 0 & 0 \\
a_{21} & a_{22} & a_{23} & 0 & 0 & 0 \\
0 & a_{32} & a_{33} & a_{34} & 0 & 0 \\
0 & 0 & 0 & a_{44} & 0 & 0 \\
a_{51} & a_{52} & a_{53} & 0 & a_{55} & 0 \\
a_{61} & 0 & a_{63} & 0 & 0 & a_{66}
\end{array}\right|
$$

where $a_{\mathrm{ij}}$ - matrix elements which depend on condition and system parameters [6].

The solution (1) looks like:

$$
\begin{aligned}
x(t) & =\sum_{1}^{n} B_{k} e^{\alpha_{k} t}+\sum_{1}^{n} 2 B_{k+1} e^{\alpha_{k+1} t} \operatorname{Sin}\left(\beta t+\phi_{i}\right) \\
& =\sum_{1}^{n}\left[M_{H}\left(\lambda_{i}\right) \cdot\left(D^{\prime}\left(\lambda_{i}\right)\right)^{-1}\right] \cdot e^{\lambda_{i} t}
\end{aligned}
$$

where $M_{H}(\lambda \mathrm{i})$ and $\left.D^{\prime}\left(\lambda_{i}\right)\right)$ - polynomials defining zeroes (numerator) and poles (denominator) of a transfer function of the investigated system [1].

For the investigated system under initial conditions: $U$ $=1, P_{d}=3, x_{c}=0.3, x_{d \Sigma}=2.3, T_{j}=7 \mathrm{~s}, T_{d 0}=2 \mathrm{~s}, T_{e}=1 \mathrm{~s}$, $T_{\mathrm{I}}=T_{U}=0.1 \mathrm{~s}, k_{0 U}=10, k_{1 U}=30, k_{0 \delta}=10, k_{1 \delta}=10$ and $\delta=700$ elements of a coefficient matrix (4) are equal to: $a_{12}=1, a_{21}=-19.0431, a_{22}=-134.5714, a_{23}=-18.3263, a_{32}$ $=2.6624, a_{33}=-1.9167, a_{34}=1.9167, a_{44}=-1, a_{45}=1, a_{52}$ $=75.1048, a_{55}=-10, a_{61}=2.5035, a_{63}=-0.9014, a_{66}=-10$.

Application of algorithm (3) to (4) gives the following secular equation:

$$
\begin{aligned}
D\left(\lambda_{i}\right)= & a_{0} \lambda_{6}+a_{1} \lambda_{5}+a_{2} \lambda_{4} \\
& +a_{3} \lambda_{3}+a_{4} \lambda_{2}+a_{5} \lambda_{1}+a_{6}=0
\end{aligned}
$$

with coefficients: $a_{0}=1, a_{1}=157.5, a_{2}=3312, a_{3}=23373$, $a_{4}=62155, a_{5}=126430, a_{6}=50859$. Thereby roots of the secular equation: $\lambda_{1}=-10,00, \lambda_{2}=-134,06, \lambda_{3}=-10,78$, $\lambda_{4,5}=-1.07 \pm j 2.41, \lambda_{6}=-0.51$. As may be inferred from structure of the roots, dominating roots have the real and complex values. It means that in the case if a system condition became heavier there are probable both non- periodic loss of stability and self-oscillation.

Transient responses and their performances can be checked up with a traditional method applying a unit step excitation and delta function to an input as a result of which action it is possible to receive transient and weight characteristics of the investigated system [1, 4]. For this purpose it is necessary to uncover a right member (6) taking into account automatic excitation control parameters and roots of a secular equation and its derivative [8-10].

Figure 1 represents the transient characteristic in the case of excitation control by a deviation and the firstorder derivative with respect to an angle.

Figure 2 shows the pulse-response characteristic of the investigated electrical power system in the case of application of a delta function. For the given automatic

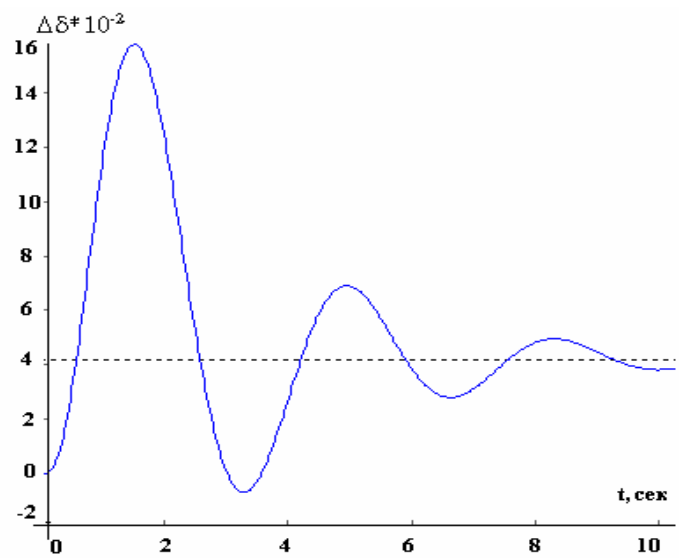

Figure 1. The transient characteristic of the elementary electrical power system in the case of availability of a strong excitation to synchronous generator with automatic excitation control: $k_{0 \delta}=50, k_{1 \delta}=10, k_{0 U}=10, k_{1 U}=30$.

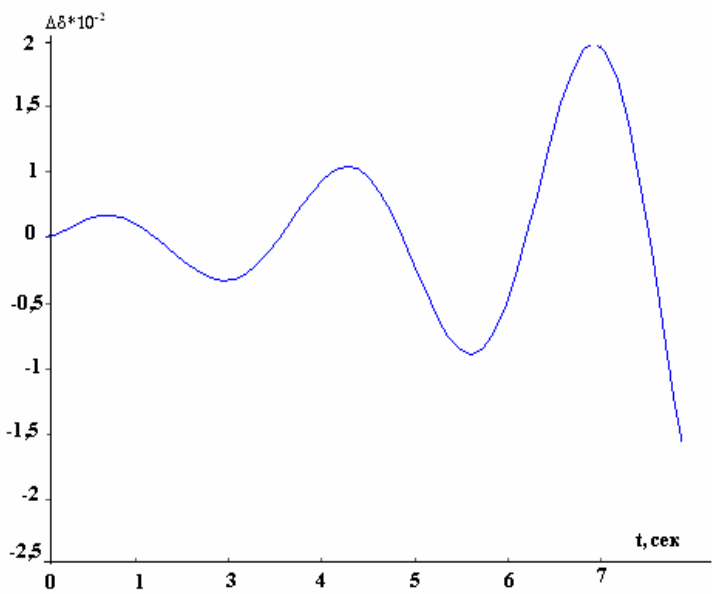

Figure 2. Pulse-response characteristic of the investigated model of an elementary electrical power system in the case of availability of a strong excitation to synchronous generator with automatic excitation control: $k_{0 \delta}=150, k_{1 \delta}=10, k_{0 U}=$ $10, k_{1 U}=30$. 
excitation control parameters the system is self-oscillating. The analysis shows that self-oscillation is removed if to select (to optimize) amplification coefficients for the first-order derivative with respect to an angle and generator voltage, provided that efficiency of the channel of a derivative with respect to an angle is essentially higher, than to the voltage channel.

\section{Conclusions}

As the analysis shows, realization of the given technique for transient response research in case of complicated electrical power system is most effective, as the secular equation is formed on the basis of a coefficient matrix for the investigated system, and the higher degree of the differential equations, the more advantage as regards to formalization and computing time consumption.

Thus, application of matrix methods for rearrangement of the equations for electrical power systems when researching a static stability of system allows making effective algorithms and their rather simple software implementation. The given technique can be applied to control of electrical power system conditions in dispatching services.

\section{REFERENCES}

[1] N. N. Ivaschenko, Automatic Control. Theory and System Elements. M: Mashinostroenie, 1973.

[2] V. A. Venikov. Transient Electromechanical Processes in
Electric Systems. M: Visshaya shkola, 1984.

[3] "Automation of Dispatching Control in Electric Power Industry," Under the editorship of Yu. N. Rudenko and V. A. Semenov, M: Izdatelstvo, 2000.

[4] P. Derusso, R. Roy and C. Claws, "State Space in the Control Theory," Under the Editorship of M. V. Meerov, Translated from the English, M: Nauka, 1970.

[5] F. R. Gantmakher, "Theory of matrices," M: Fizmatlit, 2005.

[6] K. R. Allaev and A. M. Mirzabaev, "Matrix Methods of Research of a Static Stability of Electric Systems," Article in the Issue of the Magazine.

[7] A. M. Lyapunov, "General Problem about Movement Stability,” M - L. 1950. State Publishing House Tech. theor. lit.

[8] K. R. Allaev, "The Research of Static Stability of Electric Systems by the Method of Liapunov Functions of Quadric Form T. Fan," Proceedings of the Academy of Sciences of the Republic of Uzbekistan, 1973, No.5, pp. 13-17.

[9] K. R. Allaev and A. M. Mirzabaev, "The Exploration of Static Stability of Electric Energy Systems with Joint Application of Lyapunov Function Method of Quadric Form and Nodal Equations. In book: Stability and Reliability Issues of Energy Systems. Under the editorship of L. A. Koshev," Institute of high temperatures of the USSR academy of sciences, 1990, pp. 272-276.

[10] K. R. Allaev and A. M. Mirzabaev, "To Application of the Matrix Analysis for Research of a Static Stability of Electric Systems," Problems of Energy and Resource Saving, 2009, No. 3-4, pp. 15-21. 